Homology, Homotopy and Applications, vol.20(2), 2018, pp.179-205

\title{
ON THE PATH HOMOLOGY THEORY OF DIGRAPHS AND EILENBERG-STEENROD AXIOMS
}

\author{
ALEXANDER GRIGOR'YAN, ROLANDO JIMENEZ, YURI MURANOV \\ AND SHING-TUNG YAU \\ (communicated by Alexander Mishchenko)
}

\begin{abstract}
In the paper we continue the investigation of the path homology theory of digraphs that was constructed in our previous papers. We prove basic theorems that are similar to the theorems of classical algebraic topology and introduce several natural constructions of digraphs which are very helpful to investigate the path homology theory. We describe relation of our results to the Eilenberg-Steenrod axiomatic of homology theory.
\end{abstract}

\section{Introduction}

The homology and homotopy theory of digraphs considered in this paper were introduced in $[\mathbf{1 6}, \mathbf{1 7}, \mathbf{1 8}, \mathbf{1 9}]$. Our approach is closely related to geometric and algebraic topology $[\mathbf{1 4}, \mathbf{1 3}, \mathbf{2 9}, \mathbf{2 4}, \mathbf{2 1}]$, to physical applications of graph theory $[\mathbf{3 2}, \mathbf{1 1}, \mathbf{1 0}, \mathbf{4}, \mathbf{9}]$, to Atkins homotopy theory $[\mathbf{1}, \mathbf{2}, \mathbf{3}, \mathbf{6}]$, to the theory of quivers $[\mathbf{8}, \mathbf{2 2}, \mathbf{1 5}, \mathbf{1 9}]$, and to various discrete (co)homology and homotopy theories $[\mathbf{5}, \mathbf{3}$, $6,31,12,28,23,7]$.

In the present paper we introduce a path homology theory on the category of digraph pairs (Section 3). We verify that this homology theory satisfies the properties that are analogous to Eilenberg-Steenrod axioms (Section 5). In particular, we prove that the path homologies satisfy a digraph version of the excision axiom of EilenbergSteenrod (Theorem 3.29).

Another important result is construction of the Mayer-Vietoris exact sequence for certain triads of digraphs (Theorem 3.25). This result is used, in particular, to obtain the homology groups of suspensions and wedge sums (Theorems 4.13, 4.14, Corollary 3.28).

We construct new exact sequences and a braid of exact sequence for various pairs and triples of digraphs (Theorems 3.15, 3.18, 3.21).

Note that many results of this paper can be transferred to the case of non-directed graphs, using the natural identification of the category of non-directed graphs with a full subcategory of digraphs (see [16]).

Received September 11, 2016, revised January 19, 2018; published on May 30, 2018.

2010 Mathematics Subject Classification: 05C20, 05C25, 05C38, 05C76, 18G60, 55N35, 55N40, 55P10, 55P40, 57M15.

Key words and phrases: homology of digraphs, paths in digraphs, homotopy theory for digraphs, Eilenberg-Steenrod axioms.

Article available at http://dx.doi.org/10.4310/HHA.2018.v20.n2.a9

Copyright (C) 2018, International Press. Permission to copy for private use granted. 
The paper is organized as follows. In Section 2, we give some preliminary material concerning the homotopy theory for digraphs (see also $[\mathbf{1 6}, \mathbf{1 8}]$ ) and prove several technical results.

In Section 3, we introduce and study the path homology theory for the category of digraph pairs. In particular, we construct new exact sequences of the homology groups and describe new relations between cones, suspensions, and cylinders of digraphs.

In Section 4, we introduce the natural homotopical generalizations of cylinder, cone, and suspension for digraphs and prove their properties.

In Section 5, we discuss basic properties of path homology in connection with the Eilenberg-Steenrod axioms of homology theory on topological spaces. It seems that the discrete analogue of this system of axioms does not possess completeness, and an interesting question arises of what additional axioms are needed.

In Section 6, we provide several examples which illustrate the distinction between path homology theory and homology theory for topological spaces.

\section{Acknowledgments}

The first and third authors were supported in part by SFB 1283 of the German Research Council. The second author was supported in part by the CONACyT Grant 284621. The fourth author was supported in part by the grant "Geometry and Topology of Complex Networks", no. FA-9550-13-1-0097.

\section{On homotopy theory for digraphs}

In this section we give necessary definitions and preliminary material about the homotopy theory for digraphs constructed in [16]. We also prove several technical results.

Definition 2.1. A directed graph (digraph) $X$ is a couple $\left(V_{X}, E_{X}\right)$ where $V_{X}$ is an arbitrary set whose elements are called vertices, and $E_{X} \subset\left\{V_{X} \times V_{X} \backslash \operatorname{diag}\right\}$. The elements of $E_{X}$ are called arrows (or directed edges). The fact that $(v, w) \in E_{X}$ will be denoted by $v \rightarrow w$. We write $v \rightrightarrows w$ if either $v=w$ or $v \rightarrow w$.

A based digraph $X^{*}$ is a digraph $X$ together with a fixed vertex $* \in V_{X}$.

Definition 2.2. A digraph map (or simply map) from a digraph $X$ to a digraph $Y$ is a map $f: V_{X} \rightarrow V_{Y}$ such that, for any arrow $v \rightarrow w$ in $X$ we have $f(v) \rightrightarrows f(w)$ on $Y$.

A digraph map of based digraphs $f: X^{v} \rightarrow Y^{w}$ is a digraph map of digraphs such that $f(v)=w$.

The image $f(X)$ of a digraph map $f: X \rightarrow Y$ is a digraph whose vertices are $f(v)$, $v \in V_{X}$ and whose arrows are $f(v) \rightarrow f(w)$ provided $v \rightarrow w$ and $f(v) \neq f(w)$.

A digraph map $f: X \rightarrow Y$ is called an isomorphism if $f$ is a bijection and $v \rightarrow w$ in $X$ is equivalent to $f(v) \rightarrow f(w)$ in $Y$. If there is an isomorphism $f: X \rightarrow Y$ then $X$ and $Y$ are called isomorphic. We write in this case $X \cong Y$.

Denote by $\mathcal{D}$ the category of digraphs in which the morphisms are digraph maps and by $\mathcal{D}^{*}$ the category of based digraphs and based digraph maps. 
Definition 2.3. A Cartesian product $\Pi=X \square Y$ of two digraphs $X=\left(V_{X}, E_{X}\right)$ and $Y=\left(V_{Y}, E_{Y}\right)$ is a digraph $\Pi$ with the set of vertices $V_{\Pi}=V_{X} \times V_{Y}$ and the set of arrows $E_{\Pi}$ that is defined as follows: for all $x, x^{\prime} \in V_{X}$ and $y, y^{\prime} \in V_{y}$

$$
(x, y) \rightarrow\left(x^{\prime}, y^{\prime}\right) \text { in } \Pi \quad \Longleftrightarrow \quad x^{\prime}=x, y \rightarrow y^{\prime} \quad \text { or } \quad x \rightarrow x^{\prime}, y^{\prime}=y .
$$

Let $I_{n}(n \geqslant 0)$ denote a digraph with the set of vertices $V_{I_{n}}=\{0,1, \ldots, n\}$ and the set of arrows $E_{I_{n}}$ containing exactly one of the arrows $i \rightarrow(i+1),(i+1) \rightarrow i$ for each $i=0,1, \ldots, n-1$, and no other edges. Such a digraph $I_{n}$ is called a line digraph. The number $n$ is called the height of $I_{n}$. If $I$ is a line digraph, then its height will be denoted by $|I|$. Denote by $I_{n}^{*}$ the based line digraph with base vertex $*=0$.

Denote by $\mathcal{I}_{n}$ the set of all line digraphs of height $n$, and by $\mathcal{I}_{n}^{*}$ the set of all based line digraphs of height $n$. Set also

$$
\mathcal{I}=\bigcup_{n \geqslant 0} \mathcal{I}_{n}, \quad \mathcal{I}^{*}=\bigcup_{n \geqslant 0} \mathcal{I}_{n}^{*} .
$$

Note that there is only one line digraph of height 0 , that is $\{0\}$, and two line digraphs of height $1: I=(0 \rightarrow 1)$ and $I^{-}=(0 \leftarrow 1)$.

Definition 2.4. i) We call digraph maps $f, g: X \rightarrow Y$ homotopic and write $f \simeq g$, if there exists a line digraph $I_{n} \in \mathcal{I}$ and a digraph map

$$
F: X \square I_{n} \rightarrow Y \text { such that }\left.F\right|_{X \square\{0\}}=f,\left.\quad F\right|_{X \square\{n\}}=g,
$$

where we identify $X$ with $X \square\{0\}$ and with $X \square\{n\}$ by means of the natural inclusions. If $n=1$ then we refer to $F$ as a one-step homotopy from $f$ to $g$, and the maps $f$ and $g$ will be called one-step homotopic.

ii) Two based digraph maps $f, g: X^{v} \rightarrow Y^{*}$ are called homotopic if there is a homotopy $F$ as in i) with the additional property $F\left(\{v\} \square I_{n}\right)=* \in Y$.

Now the homotopy equivalence $X \simeq Y$ of two digraphs and a contractible digraph are defined by a standard way.

Denote by Ho $\mathcal{D}$ the category of digraphs with classes of homotopic maps and by Ho $\mathcal{D}^{*}$ the category of based digraphs with classes of based homotopic maps.

Definition 2.5. i) A digraph $Y$ is called a sub-digraph of a digraph $X$ if $V_{Y} \subset V_{X}$ and $E_{Y} \subset E_{X}$. In this case we shall write $Y \sqsubset X$.

ii) If in addition

$$
v, w \in V_{Y} \text { and } v \rightarrow w \text { in } X \Longrightarrow v \rightarrow w \text { in } Y,
$$

then we say that $Y$ is an induced sub-digraph of $X$ and write $Y \subset X$.

Clearly $Y \subset X$ implies $Y \sqsubset X$. A pair $(X, Y)$ consisting of a digraph $X$ with a sub-digraph $Y \sqsubset X$ will be called a digraph pair.

Let $(X, Y)$ be a digraph pair with $Y \subset X$. Then we have the standard definition of a retraction $r: X \rightarrow Y$ and a (strong) deformation retraction $F: X \square I_{n} \rightarrow Y$ of $X$ onto $Y$.

Definition 2.6. If we have a deformation retraction $F$ with $n=1$, then we refer to $F$ as a one-step retraction. 
Note that if $Y \sqsubset X$ but $Y \not \subset X$, then there is no retraction of $X$ onto $Y$.

The following results follows directly from Definition 2.6 .

Proposition 2.7. Consider a digraph $X$ and two sub-digraphs $Y_{i} \in X(i=1,2)$ such that $X=Y_{1} \cup Y_{2}$ and there is a strong deformation retraction $F: Y_{2} \square I_{n} \rightarrow Y_{1} \cap Y_{2}$. Then there is a strong deformation retraction $\Phi: X \square I_{n} \rightarrow Y_{1}$ such that $\left.\Phi\right|_{Y_{2} \square I_{n}}=F$.

Proposition 2.8. Consider a triple of digraphs $Z \subset Y \subset X$. Let $Z$ be a retract (deformation retract, strong deformation retract) of $Y$, and let $Y$ be a retract (deformation retract, strong deformation retract) of $X$. Then $Z$ is a retract (deformation retract, strong deformation retract) of $X$.

Definition 2.9. i) Let $(X, Y)$ and $\left(X^{\prime}, Y^{\prime}\right)$ be digraph pairs. A digraph map $f: X \rightarrow$ $X^{\prime}$ is called a digraph map of pairs if $f(Y) \sqsubset Y^{\prime}$. In this case we write

$$
f:(X, Y) \rightarrow\left(X^{\prime}, Y^{\prime}\right) .
$$

ii) Two digraph maps of pairs

$$
f, g:(X, Y) \rightarrow\left(X^{\prime}, Y^{\prime}\right)
$$

are called homotopic if there exists a map of digraph pairs

$$
F:\left(X \square I_{n}, Y \square I_{n}\right) \rightarrow\left(X^{\prime}, Y^{\prime}\right),
$$

such that

$$
\left.F\right|_{(X \square\{0\}, Y \square\{0\})}=f,\left.\quad F\right|_{(X \square\{n\}, Y \square\{n\})}=g .
$$

It is easy to check that we obtain a well defined category $\mathcal{D}^{2}$ of digraph pairs and digraph maps of pairs, and a well defined category Ho $\mathcal{D}^{2}$ of digraph pairs and homotopy classes of digraph maps of pairs. Note that a based digraph $X^{*}$ can be considered as a digraph pair $(X, *)$. The based digraph map $f: X^{v} \rightarrow Y^{w}$ can be considered as a digraph map of pairs $f:(X, v) \rightarrow(Y, w)$. Thus, the category $\mathcal{D}^{*}$ is a full subcategory of $\mathcal{D}^{2}$, and similarly $\mathrm{Ho}^{*}$ is a full subcategory of $\mathrm{Ho} \mathcal{D}^{2}$.

Definition 2.10. Let $f: X \rightarrow Y$ be a digraph map. The cylinder $C_{f}$ of $f$ is a digraph with the set of vertices $V_{C_{f}}=V_{X} \cup V_{Y}$ and with the set of arrows that consists of all arrows of $X$, all arrows of $Y$, and of all arrows of the form $v \rightarrow f(v), v \in V_{X}$.

The inverse cylinder $C_{f}^{-}$has the same set of vertices as $C_{f}$ and in the definition of the set of arrows $v \rightarrow f(v)$ should be replaced by $v \leftarrow f(v)$.

In particular, we have natural inclusions $j: X \rightarrow C_{f}$ and $i: Y \rightarrow C_{f}$, and similarly for $C_{f}^{-}$. For example, for $f=\operatorname{Id}_{X}: X \rightarrow X$ we obtain digraphs

$$
C_{f} \cong X \square I \cong C_{f}^{-} \cong X \square I^{-},
$$

that are called cylinders over the digraph $X$.

Definition 2.11. The cylinder of the map $X \rightarrow\{a\}$ of a digraph $X$ to a single vertex digraph $\{a\}$ is called a cone over the digraph $X$ and is denoted by $C_{0 n e}(X)$ or simply by Cone $(X)$. The inverse cylinder of this map is called an inverse cone over the digraph $X$ and is denoted $C_{\text {Cone }}^{-}(X)$ or simply by $\operatorname{Cone}^{-}(X)$. 
Definition 2.12. The suspension $S(X)$ of a digraph $X$ is a digraph defined as the union

$$
\operatorname{Cone}_{a}(X) \bigcup_{X \sim X} \operatorname{Cone}_{b}(X)
$$

of two cones $C_{\text {Cone }}(X)$ and $C_{a n e}(X)$ where $a \neq b$, and we identify $X \subset C_{\text {Cone }}(X)$ with $X \subset C \operatorname{Cone}_{b}(X)$. Similarly, the inverse suspension $S^{-}(X)$ is the union of Cone $_{a}^{-}(X)$ and Cone $_{b}^{-}(X)$.

Definition 2.13. Let $f: X \rightarrow Y$ be a digraph map. The cone of $f$ is a digraph Cone $_{f}$ whose set of vertices is $V_{\text {Cone }_{f}}=V_{Y} \cup\{*\}$ and whose set of arrows consists of all the arrows of $Y$ and all arrows $* \rightarrow f(v), v \in V_{X}$. Similarly one defines the inverse cone of $f$ where $* \rightarrow f(v)$ is replaced by $* \leftarrow f(v)$.

For example, for an identity map $f=\operatorname{Id}_{X}: X \rightarrow X$, we have

$$
\text { Cone }_{f} \cong \text { Cone }^{-}(X)
$$

and

$$
\operatorname{Cone}_{f}^{-} \cong \operatorname{Cone}(X) \text {. }
$$

Definition 2.14. i) For any $n \geqslant 0$ define a digraph $\Delta^{n}$ by

$$
V_{\Delta^{n}}=\{0,1, \ldots, n\}, \quad i \rightarrow j \text { in } \Delta^{n} \Leftrightarrow i<j .
$$

An $n$-simplex is any digraph that is isomorphic to $\Delta^{n}$.

ii) For any $n \geqslant 0$, define a digraph $I^{n}$ as

$$
I^{0}=\{*\}, \quad I^{n}=\underbrace{I \square \cdots \square I}_{n \text { times }} .
$$

An $n$-cube is any digraph that is isomorphic to $I^{n}$.

Proposition $2.15([\mathbf{1 6}])$. i) For any digraph $X$ and for any line digraph $I_{n}$ there are homotopy equivalences:

$$
X \square I_{n} \simeq X, \quad \text { Cone }(X) \simeq \operatorname{Cone}^{-}(X) \simeq\{*\} .
$$

ii) For any digraph map $f: X \rightarrow Y$ we have homotopy equivalences

$$
\mathrm{C}_{f} \simeq Y \simeq \mathrm{C}_{f}^{-} \text {. }
$$

iii) For any $n \geqslant 0$, we have homotopy equivalences

$$
I^{n} \simeq\{*\} \simeq \Delta^{n}
$$

Now we state and prove yet several results about homotopy properties of digraphs that will be needed in the following sections.

For an $n$-simplex $\Delta^{n}(n \geqslant 1)$ and for any $0 \leqslant k \leqslant n$, define its $k$-face as a subdigraph $\Delta_{k}^{n-1}$ obtained from $\Delta^{n}$ by deleting one vertex $k \in V_{\Delta^{n}}$ and all arrows that are incident to $k$.

Recall that the $n$-cube $I^{n}$ can be described as a digraph of $2^{n}$ vertices such that any vertex $\alpha \in V_{I^{n}}$ can be identified with a sequence $\alpha=\left(a_{1}, \ldots, a_{n}\right)$ of binary digits so that $\alpha \rightarrow \beta$ in $I_{n}$ if and only if the sequence $\beta=\left(b_{1}, \ldots, b_{n}\right)$ is obtained from $\alpha=\left(a_{1}, \ldots, a_{n}\right)$ by replacing a digit 0 by 1 at exactly one position. 
Let $I^{n}$ be an $n$-cube digraph with $n \geqslant 1$. For any $1 \leqslant p \leqslant n$ we have two inclusions $I^{n-1}$ to sub-digraphs $I_{p 0}^{n-1}$ and $I_{p 1}^{n-1}$ of $I^{n}$, that are defined in the following way. For $I^{1}=0 \rightarrow 1$, we have the inclusions

$$
V_{10}^{0}:\{0\} \rightarrow I_{10}^{0}=\{0\} \subset I^{1}
$$

and

$$
V_{11}^{0}:\{0\} \rightarrow I_{11}^{0}=\{1\} \subset I^{1} .
$$

For $n \geqslant 2$ the inclusions on the sub-digraphs

$$
V_{p 0}^{n-1}: I^{n-1} \rightarrow I_{p 0}^{n-1} \subset I^{n} \text { and } V_{p 1}^{n-1}: I^{n-1} \rightarrow I_{p 1}^{n-1} \subset I^{n}
$$

are given by

$$
V_{p \epsilon}^{n-1}\left(i_{1}, \ldots, i_{n-1}\right)=\left(i_{1}, \ldots, i_{p-1}, \epsilon, i_{p}, \ldots, i_{n-1}\right)
$$

where $\epsilon=0,1$. We shall call the images $I_{p \epsilon}^{n-1} \subset I^{n}$ of the maps $V_{p \epsilon}^{n-1}$ for $\epsilon=0,1$ the first and second $p$-faces of the digraph $I_{n}$, respectively.

Proposition 2.16. Let $Y$ be a digraph.

i) Let $X=Y \cup \Delta^{n}$ and $Y \cap \Delta^{n}=\Delta_{k}^{n-1}$ where $n \geqslant 1,0 \leqslant k \leqslant n$. Then there is a one-step strong deformation retraction $F: X \square I \rightarrow X$ of $X$ onto $Y$.

ii) Let $X=Y \cup I^{n}$ and $Y \cap I^{n}=I_{p \epsilon}^{n-1}$ where $n \geqslant 1,0 \leqslant p \leqslant n$. Then there is a one-step strong deformation retraction $F: X \square I \rightarrow X$ of $X$ onto $Y$.

Proof. i) Define a strong deformation retraction of $\Delta^{n}$ to $\Delta_{k}^{n-1}$ for two different cases of $k$. In the case $k \neq n$ define $F: \Delta^{n} \square I \rightarrow \Delta^{n}$ on the set of vertices as

$$
F(v, i)= \begin{cases}v, & \text { for } i=0 \\ v, & \text { for } i=1, v \neq k \\ k+1, & \text { for } i=1, v=k\end{cases}
$$

In the case $k=n$ define $F: \Delta^{n} \square I^{-} \rightarrow \Delta^{n}$ on the set of vertices as

$$
F(v, i)= \begin{cases}v, & \text { for } i=0 \\ v, & \text { for } i=1, v \neq n \\ n-1, & \text { for } i=1, v=n\end{cases}
$$

Now statement i) follows from Proposition 2.7.

ii) Consider the case $\epsilon=1$. Define a strong deformation retraction $F: I^{n} \square I \rightarrow I^{n}$ of $I^{n}$ to $I_{p 1}^{n-1}$ on the set of vertices as

$$
F\left(\left(i_{1}, \ldots, i_{n}\right), i\right)= \begin{cases}\left(i_{1}, \ldots, i_{n}\right), & \text { for } i=0, \\ \left(i_{1}, \ldots, i_{n}\right), & \text { for } i=1, i_{p}=1 \\ \left(i_{1}, \ldots, i_{p-1}, 1, i_{p}, \ldots, i_{n}\right), & \text { for } i=1, i_{p}=0\end{cases}
$$

Consider the case $\epsilon=0$. Define a strong deformation retraction $F: I^{n} \square I^{-} \rightarrow I^{n}$ of 
$I^{n}$ to $I_{p 0}^{n-1}$ on the set of vertices as

$$
F\left(\left(i_{1}, \ldots, i_{n}\right), i\right)= \begin{cases}\left(i_{1}, \ldots, i_{n}\right), & \text { for } i=0, \\ \left(i_{1}, \ldots, i_{n}\right), & \text { for } i=1, i_{p}=0, \\ \left(i_{1}, \ldots, i_{p-1}, 0, i_{p}, \ldots, i_{n}\right), & \text { for } i=1, i_{p}=1 .\end{cases}
$$

Now statement ii) follows from Proposition 2.7.

Proposition 2.17. Let $Y_{1} \subset Y$ be a digraph pair. Assume that there is a strong deformation retraction $F: Y \square I_{n} \rightarrow Y$ of $Y$ to $Y_{1}$. Then, for any digraph $X$, there is a strong deformation retraction $\operatorname{Id} \square F: X \square\left(Y \square I_{n}\right) \rightarrow X \square Y$ of $X \square Y$ to $X \square Y_{1}$.

Proof. The digraph homomorphism Id $\square F$ is a deformation retraction.

Corollary 2.18. Let $K=I^{n} \square \Delta^{m}$. Assume that $K_{1}$ is a sub-digraph of $K$ that is equal to $I^{n} \square \Delta_{m}^{n-1}$ or $I_{p \epsilon}^{n-1}$, where $\Delta_{m}^{n-1} \subset \Delta^{n}$ and $I_{p \epsilon}^{n-1} \subset I^{n}$ are the faces of the cube and simplex, respectively. Then there is a strong deformation retraction $F: K \square I \rightarrow K$ of $K$ to $K_{1}$.

\section{Path homology of digraphs and digraphs pairs}

In this section we recall the definition of path homology for a finite digraph $X=(V, E)$ with coefficients in an arbitrary abelian group $K$ (see [16] and [18]) and introduce the path homology theory for the category of digraph pairs. Then we construct new exact sequences of the homology groups and describe new relations between cones, suspensions, and cylinders of digraphs.

Let $G=(V, E)$ be a digraph. At first, we define a chain complex $\Lambda_{*}(V, K)$ of arbitrary paths on the set $V$ of vertices. Then, using a digraph structure given by arrows, we define a chain complex of digraph $G$ and the notion of homology of digraphs (see [16] and [18]). This approach is dual to the construction of the cochain complex and cohomology groups of digraphs that is based on the universal calculus on the algebra of functions on the vertices [17].

Definition 3.1. For any non-negative integer $p$, an elementary p-path on the set $V$ of vertices is any sequence $\left\{i_{k}\right\}_{k=0}^{p}$ of $p+1$ vertices of $V$. For $p=-1$, an elementary $p$-path is the empty set $\emptyset$.

The elementary $p$-path $\left\{i_{k}\right\}_{k=0}^{p}$ will also be denoted simply by $i_{0} \ldots i_{p}$, without delimiters between the vertices.

Denote by $\Lambda_{p}=\Lambda_{p}(V, K)$ the abelian group that consists of all formal $K$-linear combinations of all elementary $p$-paths.

Definition 3.2. The elements of $\Lambda_{p}$ are called p-paths on $V$.

An elementary $p$-path $i_{0} \ldots i_{p}$ as an element of $\Lambda_{p}$ will be denoted by $e_{i_{0} \ldots i_{p}}$. The empty set as an element of $\Lambda_{-1}$ will be denoted by $e$. By definition, the set $\left\{e_{i_{0} \ldots i_{p}} \mid i_{0}, \ldots, i_{p} \in V\right\}$ is a basis of $\Lambda_{p}$. Each $p$-path $v$ can be presented by a unique way as a finite sum

$$
v=\sum_{i_{0}, \ldots, i_{p} \in V} v^{i_{0} \ldots i_{p}} e_{i_{0} \ldots i_{p}}
$$

where $v^{i_{0} \cdots i_{p}} \in K$. Note that $\Lambda_{-1}$ consists of all multiples of $e$, so that $\Lambda_{-1} \cong K$. 
Definition 3.3. For any $p \geqslant 0$, define a homomorphism $\partial: \Lambda_{p} \rightarrow \Lambda_{p-1}$ on the elementary paths

$$
\partial e_{i_{0} \ldots i_{p}}=\sum_{q=0}^{p}(-1)^{q} e_{i_{0} \ldots \widehat{i_{q}} \ldots i_{p}}
$$

(where $\widehat{i_{q}}$ means omission of the index $i_{q}$ ) and then extend $\partial$ to $\Lambda_{p}$ by $K$-linearity. We shall refer to $\partial$ as the boundary operator.

For an arbitrary $p$-path (1) with $p \geqslant 0$, we have

$$
\partial v=\sum_{i_{0}, \ldots, i_{p}} v^{i_{0} \ldots i_{p}} \partial e_{i_{0} \ldots i_{p}}=\sum_{i_{0}, \ldots, i_{p}} \sum_{q=0}^{p}(-1)^{q} v^{i_{0} \ldots i_{p}} e_{i_{0} \ldots \widehat{i_{q}} \ldots i_{p}},
$$

whence

$$
\begin{aligned}
(\partial v)^{j_{0} \ldots j_{p-1}} & =\sum_{i_{0}, \ldots, i_{p}} \sum_{q=0}^{p}(-1)^{q} v^{i_{0} \ldots i_{p}}\left(e_{i_{0} \ldots \widehat{i_{q}} \ldots i_{p}}\right)^{j_{0} \ldots j_{p-1}} \\
& =\sum_{k \in V} \sum_{q=0}^{p}(-1)^{q} v^{j_{0} \ldots j_{q-1} k j_{q} \ldots j_{p-1}}
\end{aligned}
$$

where the index $k$ is inserted in the path $j_{0} \ldots j_{p-1}$ between $j_{q-1}$ and $j_{q}$ if $1 \leqslant q<p$, before $j_{0}$ if $q=0$, and after $j_{p-1}$ if $q=p$.

Set also $\Lambda_{-2}=\{0\}$ and define $\partial: \Lambda_{-1} \rightarrow \Lambda_{-2}$ to be zero.

Lemma 3.4. We have $\partial^{2}=0$.

Proof. The operator $\partial^{2}$ acts from $\Lambda_{p}$ to $\Lambda_{p-2}$, so that the identity $\partial^{2}=0$ makes sense for all $p \geqslant 0$. In the case $p=0$ the identity $\partial^{2}=0$ is trivial. For $p \geqslant 1$, we have by (1)

$$
\begin{aligned}
\partial^{2} e_{i_{0} \ldots i_{p}} & =\sum_{q=0}^{p}(-1)^{q} \partial e_{i_{0} \ldots \widehat{i_{q}} \ldots i_{p}} \\
& =\sum_{q=0}^{p}(-1)^{q}\left(\sum_{r=0}^{q-1}(-1)^{r} e_{i_{0} \ldots \widehat{i_{r} \ldots} \hat{i_{q} \ldots i_{p}}}+\sum_{r=q+1}^{p}(-1)^{r-1} e_{i_{0} \ldots \widehat{i_{q} \ldots} \hat{i_{r} \ldots i_{p}}}\right) \\
& =\sum_{0 \leqslant r<q \leqslant p}(-1)^{q+r} e_{i_{0} \ldots \widehat{r_{r} \ldots} \ldots \hat{i_{q} \ldots i_{p}}}-\sum_{0 \leqslant q<r \leqslant p}(-1)^{q+r} e_{i_{0} \ldots \widehat{i_{q}} \ldots \widehat{i_{r} \ldots i_{p}}} .
\end{aligned}
$$

After switching $q$ and $r$ in the last sum we see that the two sums cancel out, whence $\partial^{2} e_{i_{0} \ldots i_{p}}=0$. This implies $\partial^{2} v=0$ for all $v \in \Lambda_{p}$.

Consequently, we have the following chain complex:

$$
0 \leftarrow K \leftarrow \Lambda_{0} \leftarrow \cdots \leftarrow \Lambda_{p-1} \leftarrow \Lambda_{p} \leftarrow \cdots,
$$

where the arrows are given by the boundary operator $\partial$.

Definition 3.5. We say that an elementary path $e_{i_{0} \ldots i_{p}}(p \geqslant 1)$ is non-regular if $i_{k-1}=i_{k}$ for some $k=1, \ldots, p$, and regular otherwise. 
For $p \geqslant 1$, consider the subgroup $I_{p}$ of $\Lambda_{p}$ spanned by non-regular elementary paths:

$$
I_{p}=I_{p}(V)=\operatorname{span}\left\{e_{i_{0} \ldots i_{p}} \mid i_{0} \ldots i_{p} \text { is non-regular }\right\} .
$$

We set also $I_{0}=I_{-1}=I_{-2}=0$.

Lemma 3.6. If $e_{i_{0} \ldots i_{p}} \in I_{p}(p \geqslant 1)$ then $\partial e_{i_{0} \ldots i_{p}} \in I_{p-1}$ and, putting $\mathcal{R}_{p}:=\Lambda_{p} / I_{p}$, we obtain the chain factor complex

$$
0 \leftarrow K \leftarrow \mathcal{R}_{0} \leftarrow \cdots \leftarrow \mathcal{R}_{p-1} \leftarrow \mathcal{R}_{p} \leftarrow \cdots
$$

Proof. For an $e_{i_{0} \ldots i_{p}} \in I_{p}$ there exists an index $k$ such that $i_{k}=i_{k+1}$. Then we have

$$
\begin{aligned}
\partial e_{i_{0} \ldots i_{p}}= & e_{i_{1} \ldots i_{p}}-e_{i_{0} i_{2} \ldots i_{p}}+\cdots \\
& +(-1)^{k} e_{i_{0} \ldots i_{k-1} i_{k+1} i_{k+2} \ldots i_{p}}+(-1)^{k+1} e_{i_{0} \ldots i_{k-1} i_{k} i_{k+2} \ldots i_{p}} \\
& +\cdots+(-1)^{p} e_{i_{0} \ldots i_{p-1}} .
\end{aligned}
$$

By $i_{k}=i_{k+1}$ the two terms in the middle line of (4) cancel out, whereas all other terms are non-regular, whence $\partial e_{i_{0} \ldots i_{p}} \in I_{p-1}$.

Note that the chain complex $\mathcal{R}_{*}$ in $(3)$ is defined for a finite set $V$. Thus we can denote it by $\mathcal{R}_{*}(V)$, and similarly $\Lambda_{p}(V)$ and $I_{p}(V)$ are defined. Any map of finite sets $f: V \rightarrow V^{\prime}$ defines for any $p \geqslant 0$ the induced homomorphism

$$
f_{*}: \Lambda_{p}(V) \rightarrow \Lambda_{p}\left(V^{\prime}\right)
$$

by the rule $f_{*}\left(e_{i_{0} \ldots i_{p}}\right)=e_{f\left(i_{0}\right) \ldots f\left(i_{p}\right)}$, extended to $\Lambda_{p}(V)$ by linearity. The map $f_{*}$ can be considered as a morphism of chain complexes, because by $(2) \partial f_{*}=f_{*} \partial$. Since $f_{*}\left(I_{p}(V)\right) \subset I_{p}\left(V^{\prime}\right)$, we obtain the induced morphism of chain complexes (see [16])

$$
\mathcal{R}_{*}(V) \rightarrow \mathcal{R}_{*}\left(V^{\prime}\right),
$$

which we also denote by $f_{*}$. We can define this homomorphism on basic elements by the rule

$$
f_{*}\left(e_{i_{0} \ldots i_{p}}\right)= \begin{cases}e_{f\left(i_{0}\right) \ldots f\left(i_{p}\right)}, & \text { if } e_{f\left(i_{0}\right) \ldots f\left(i_{p}\right)} \text { is regular, } \\ 0, & \text { if } e_{f\left(i_{0}\right) \ldots f\left(i_{p}\right)} \text { is non-regular. }\end{cases}
$$

Now consider a digraph $X=(V, E)$.

Definition 3.7. An elementary regular $p$-path $e_{i_{0} \ldots i_{p}}$ on $X$ is called allowed if $i_{k} \rightarrow$ $i_{k+1}$ for any $k=0, \ldots, p-1$, and non-allowed otherwise.

For $p \geqslant 0$, let $\mathcal{A}_{p}(X)$ denote a subgroup of $\mathcal{R}_{p}(X):=\mathcal{R}_{p}(V)$ spanned by the allowed elementary $p$-paths. We put $\mathcal{A}_{-1}(X)=K$. The elements of $\mathcal{A}_{p}(X)$ are called allowed $p$-paths. Note that $\mathcal{A}_{p}(X)=\mathcal{R}_{p}(X)$ for $p=-1,0,1$.

Consider the following subgroup of $\mathcal{A}_{p}(X)$

$$
\Omega_{p} \equiv \Omega_{p}(X):=\left\{v \in \mathcal{A}_{p}: \partial v \in \mathcal{A}_{p-1}\right\}
$$

that is $\partial$-invariant. Indeed, $v \in \Omega_{p}$ implies $\partial v \in \mathcal{A}_{p-1}$ and $\partial(\partial v)=0 \in \mathcal{A}_{p-2}$, whence $\partial v \in \Omega_{p-1}$. The elements of $\Omega_{p}$ are called $\partial$-invariant $p$-paths. 
Hence, for any digraph $X$ we obtain a chain complex of abelian groups

$$
0 \longleftarrow K \stackrel{\partial}{\longleftarrow} \Omega_{0} \stackrel{\partial}{\longleftarrow} \Omega_{1} \stackrel{\partial}{\longleftarrow} \cdots \stackrel{\partial}{\longleftarrow} \Omega_{p-1} \stackrel{\partial}{\longleftarrow} \Omega_{p} \stackrel{\partial}{\longleftarrow} \cdots,
$$

which we shall denote by $\widetilde{\Omega}_{*}=\widetilde{\Omega}_{*}(X)=\widetilde{\Omega}_{*}(X, K)$. By construction we have $\Omega_{-1}=$ $K, \Omega_{0}=\mathcal{R}_{0}(X)$ and $\Omega_{1}=\mathcal{R}_{1}(X)$, while in general

$$
\Omega_{p} \subset \mathcal{A}_{p}(X) \subset \mathcal{R}_{p}(X) .
$$

We shall call the chain complex (6) the reduced path chain complex of the digraph $X$. Its homology groups are denoted by $\widetilde{H}_{*}(X, K)$ and are referred to as the reduced path homologies of the digraph $X$ with coefficients from $K$. We consider also the following part of the chain complex (6)

$$
0 \leftarrow \Omega_{0} \leftarrow \cdots \leftarrow \Omega_{n-1} \leftarrow \Omega_{n} \leftarrow \Omega_{n+1} \leftarrow \cdots,
$$

where the definition of the boundary operator $\partial$ on $\Omega_{0}$ is modified by setting $\partial \equiv 0$. Note that $(7)$ is a chain complex and our modification does not affect $\partial$ on $\Omega_{n}$ with $n \geqslant 1$. We shall denote this chain complex by $\Omega_{*}(X)$. The homology groups of $\Omega_{*}(X)(7)$ are referred to as the path homology groups of the digraph $X$ and are denoted by $H_{*}(X)=H_{*}(X, K), n \geqslant 0$. Let us note that the homology groups $H_{p}(X)$ (as well as the groups $\Omega_{p}(X)$ ) can be computed directly by definition using simple tools of linear algebra, in particular, those implemented in modern computational software.

Theorem $3.8([\mathbf{1 6}])$. i) Let $f: X \rightarrow X^{\prime}$ be a digraph map. Then the map $f_{*}: \mathcal{R}_{*}(V) \rightarrow$ $\mathcal{R}_{*}\left(V^{\prime}\right)$ in (5) satisfies the condition

$$
f_{*}\left(\mathcal{A}_{p}(X)\right) \subset \mathcal{A}_{p}\left(X^{\prime}\right)
$$

and its restriction $\left.f_{*}\right|_{\Omega_{p}(X)}$ provides a morphism of reduced chain complexes

$$
\widetilde{\Omega}_{*}(X, K) \rightarrow \widetilde{\Omega}_{*}\left(X^{\prime}, K\right)
$$

and, consequently, a homomorphism of reduced homology groups

$$
\widetilde{H}_{*}(X, K) \rightarrow \widetilde{H}_{*}\left(X^{\prime}, K\right)
$$

that will also be denoted by $f_{*}$. The same is true for the non-reduced complex and its homology groups.

ii) The homotopy equivalent digraph maps $f, g: X \rightarrow X^{\prime}$ induce the equal homomorphisms of (reduced) homology groups. Hence, the (reduced) homology groups introduced above are homotopy invariant.

Now we develop the path homology theory for the category of digraphs pairs. Let $Y \sqsubset X$ be a sub-digraph of a digraph $X$ and denote by $i$ the natural inclusion.

Proposition 3.9. The induced by $i$ morphism of reduced chain complexes

$$
i_{*}: \widetilde{\Omega}_{*}(Y) \rightarrow \widetilde{\Omega}_{*}(X)
$$

is an inclusion. 
Proof. As follows directly from the definition $\Lambda_{*}(Y) \subset \Lambda_{*}(X)$ and $\mathcal{R}_{*}(Y) \subset \mathcal{R}_{*}(X)$. We have $\mathcal{A}_{-1}(Y)=\mathcal{A}_{-1}(X)=K$ and

$$
\mathcal{A}_{0}(Y)=\operatorname{span}\left\{v \mid v \in V_{G}\right\} \subset \operatorname{span}\left\{v \mid v \in V_{X}\right\}=\mathcal{A}_{0}(X) .
$$

For any $p \geqslant 1$ we have the natural inclusion $\mathcal{A}_{p}(Y) \subset \mathcal{A}_{p}(X)$ since $i_{k-1} \rightarrow i_{k}$ in $Y$ implies $i_{k-1} \rightarrow i_{k}$ in $X$. Hence

$$
\left[v \in \Omega_{p}(Y) \subset \mathcal{A}_{p}(Y) \subset \mathcal{A}_{p}(X)\right] \Rightarrow\left[\partial v \in \mathcal{A}_{p-1}(Y) \subset \mathcal{A}_{p-1}(X)\right]
$$

and $\widetilde{\Omega}_{*}(Y) \subset \widetilde{\Omega}_{*}(X)$.

Note that the inclusion map $i_{*}$ in Proposition 3.9 is the identity map $K \rightarrow K$ in dimension -1 , so that $\widetilde{\Omega}_{-1}(X) / \widetilde{\Omega}_{-1}(Y)=0$. Now we define a chain complex $\Omega_{*}(X, Y)$ as factor-complex $\Omega_{*}(X) / \Omega_{*}(Y)$ that coincides with $\widetilde{\Omega}_{*}(X) / \widetilde{\Omega}_{*}(Y)$. The homology groups of this complex are denoted by $H_{*}(X, Y)=H_{*}(X, Y ; K)$ and are called the relative path homology groups.

Let us give a graph interpretation of the factor-complex in a special case. If $U$ is a subset of $V_{X}$ then denote by $\mathcal{A}_{p}^{U}(X)$ the subgroup of $\mathcal{A}_{p}(X)$ spanned by all the elementary paths $e_{i_{0} \ldots i_{p}}$ that intersect $U$ (where the latter means that at least one of the vertices $i_{0} \ldots i_{p}$ belongs to $\left.U\right)$. Set also

$$
\Omega_{p}^{U}(X)=\Omega_{p}(X) \cap \mathcal{A}_{p}^{U}(X) .
$$

Lemma 3.10. Let $Y \sqsubset X$ and let $U=V_{X} \backslash V_{Y}$. If there are no arrows from $U$ to $V_{Y}$ then

$$
\Omega_{p}(X) / \Omega_{p}(Y) \cong \Omega_{p}^{U}(X) .
$$

Proof. Any $x \in \Omega_{p}(X)$ is a linear combination of elementary paths:

$$
x=\sum_{i_{0} \ldots i_{p} \in V_{X}} x^{i_{0} \ldots i_{p}} e_{i_{0} \ldots i_{p}} .
$$

Each term $x^{i_{0} \ldots i_{p}} e_{i_{0} \ldots i_{p}}$ with non-zero $x^{i_{0} \ldots i_{p}}$ will be called an elementary term of $x$. Denote by $u$ the sum of all the elementary terms of $x$ that intersect $U$, and by $v$ the rest of $x$, that is the sum of the elementary terms of $x$ that lie in $Y$. Hence, $x=u+v$. Since $x$ is allowed, both $u$ and $v$ are allowed. Since $x$ is $\partial$-invariant, the sum $\partial u+\partial v=\partial x$ is allowed. Let $c e_{i_{0} \ldots i_{p}}$ be an elementary term of $u$. Since $e_{i_{0} \ldots i_{p}}$ is allowed and there is no arrow from $U$ to $Y$, we see that the sequence $i_{0} \ldots i_{p}$ looks as follows: some first part, say $i_{0} \ldots i_{q}$ lies in $Y$, and the rest $i_{q+1} \ldots i_{p}$ lies in $U$, where the second part is non-empty, in particular, $i_{p} \in U$. Then all the elementary terms of $\partial e_{i_{0} \ldots i_{p}}$ intersect $U$ except for one case: if $i_{p}$ is the only vertex of $e_{i_{0} \ldots i_{p}}$ in $U$ then the elementary term $e_{i_{0} \ldots i_{p-1}}$ of $\partial e_{i_{0} \ldots i_{p}}$ does not intersect $U$. Note that in the latter case $e_{i_{0} \ldots i_{p-1}}$ is an allowed path in $Y$. Hence, we obtain

$$
\partial u=(\text { allowed path in } Y)+(\text { sum of terms intersecting } U) \text {. }
$$

Since

$$
\partial v=\text { sum of terms lying in } Y,
$$

and $\partial u+\partial v$ is allowed, we see that the sum of the terms of $\partial u$ intersecting $U$ must 
be allowed, that is,

$$
\partial u=(\text { allowed path in } Y)+(\text { allowed path in } U) .
$$

Hence, $\partial u$ is allowed. It follows that $\partial v=\partial x-\partial u$ is also allowed. Hence, both $u$ and $v$ are $\partial$-invariant, consequently, $u \in \Omega_{p}^{U}(X)$ and $v \in \Omega_{p}(Y)$.

Therefore, the decomposition $x=u+v$ determines a homomorphism

$$
\begin{aligned}
\Omega_{p}(X) & \rightarrow \Omega_{p}^{U}(X), \\
x & \mapsto u,
\end{aligned}
$$

which is obviously an epimorphism. Clearly, its kernel is $\Omega_{p}(Y)$, which implies (8).

Now we transfer to path homology groups the standard results from algebraic topology (see, for example, [20, Chpt. 2.1] and [27, Chpt. 1.3]).

Theorem 3.11. Let $Y \sqsubset X$ be a sub-digraph of a digraph $X$. Then there is a relative homology long exact sequence

$$
0 \leftarrow H_{0}(X, Y) \leftarrow H_{0}(X) \leftarrow H_{0}(Y) \leftarrow \cdots \leftarrow H_{n-1}(Y) \leftarrow H_{n}(X, Y) \leftarrow H_{n}(X) \leftarrow \cdots
$$

in which the homomorphisms $H_{n}(Y) \rightarrow H_{n}(X)$ are induced by the inclusion $i: Y \rightarrow$ $X$. In particular, if the inclusion $Y \rightarrow X$ is a homotopy equivalence, then $H_{n}(X, Y)=$ 0 for all $n \geqslant 0$.

Proof. By definition, we have the short exact sequences of chain complexes

$$
0 \rightarrow \Omega_{*}(Y) \rightarrow \Omega_{*}(X) \rightarrow \Omega_{*}(X, Y) \rightarrow 0
$$

from which the result follows by standard arguments.

As follows from our construction, there is also a long exact sequence of reduced homology groups for a pair $(X, Y)$ that has exactly the same form and the groups $\widetilde{H}_{n}(X, Y)$ are the same as $H_{n}(X, Y)$.

Proposition 3.12. Let $X$ be a connected digraph. Then $H_{0}(X)=K$. For any digraph $X$ we have $H_{0}(X) \cong \oplus_{n} K$ where $n$ is the number of connectivity components of $X$.

Proof. Consider a connected digraph $X$. We can join any two vertices $v, w \in V_{X}$ by a sequence of edges $v=i_{0} \sim v_{1} \sim \cdots \sim i_{n}=w$ where $i_{k} \sim i_{k+1}$ means that at least one of the cases $i_{k} \rightarrow i_{k+1}$ or $i_{k} \leftarrow i_{k+1}$ is realized. In $\Omega_{0}(X)$ we have

$$
e_{i_{n}}=e_{i_{0}}+\partial\left(\sum_{k=0,\left(i_{k} \rightarrow i_{k+1}\right)}^{n-1} e_{i_{k} i_{k+1}}-\sum_{k=0,\left(i_{k} \leftarrow i_{k+1}\right)}^{n-1} e_{i_{k+1} i_{k}}\right),
$$

and hence any element from $\Omega_{0}(X)$ has the form $e_{i_{0}}+\operatorname{Im} \partial$. The element $e_{i_{0}}$ does not lie in the image $\partial$, since for any $x=\sum_{k} a_{k} e_{i_{k} j_{k}} \in \Omega_{1}(X)$ we have

$$
\partial\left(\sum_{k} a_{k} e_{i_{k} j_{k}}\right)=\sum_{k} a_{k} e_{j_{k}}-\sum_{k} a_{k} e_{e_{k}}
$$

where the sum of coefficients in the right part is zero. The general case can be considered in a similar way. 
Corollary 3.13. Let $X$ be a connected digraph, and $Y \sqsubset X$ a non-empty connected sub-digraph of $X$. Then $H_{0}(X, Y)=0$.

Proof. Follows from Theorem 3.11 and Proposition 3.12.

Proposition 3.14. For a digraph $X$ we have

$$
H_{n}(X)= \begin{cases}\widetilde{H}(X), & n \geqslant 1 \\ \widetilde{H}_{0}(X) \oplus K, & n=0\end{cases}
$$

Proof. The proof is standard (see, for example, [20] and [27]).

Theorem 3.15. A map $f:(X, Y) \rightarrow\left(X^{\prime}, Y^{\prime}\right)$ of digraph pairs induces a homomorphism $f_{*}: H_{n}(X, Y) \rightarrow H_{n}\left(X^{\prime}, Y^{\prime}\right)(n \geqslant 0)$ fitting into a commutative diagram

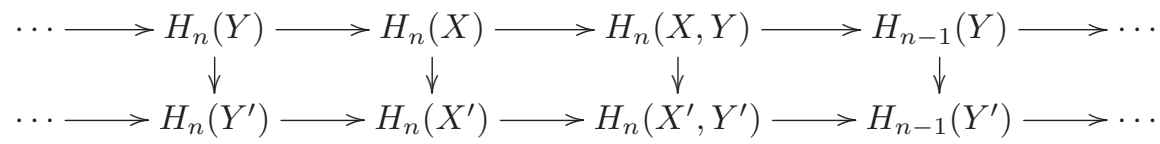

in which the rows are the relative long homology exact sequences.

Proof. Follows from [25, Chpt. 2, 4].

Theorem 3.16. i) Let two digraph maps $f, g:(X, Y) \rightarrow\left(X^{\prime}, Y^{\prime}\right)$ be homotopic as digraph maps of pairs (see Definition 2.9). Then $f_{*}=g_{*}: H_{n}(X, Y) \rightarrow H_{n}\left(X^{\prime}, Y^{\prime}\right)$ for any $n \geqslant 0$.

ii) Let a digraph map $f:(X, Y) \rightarrow\left(X^{\prime}, Y^{\prime}\right)$ be such that its restrictions $\left.f\right|_{X}$ and $\left.f\right|_{Y}$ are homotopy equivalences. Then $f_{*}: H_{n}(X, Y) \rightarrow H_{n}\left(X^{\prime}, Y^{\prime}\right)$ is an isomorphism for any $n \geqslant 0$.

Proof. Follows from the Five Lemma [25, Chpt. 1, 3] and Theorem 3.8.

Proposition 3.17. Let $* \subset X$ be the one-vertex sub-digraph of a digraph $X$. Then

$$
H_{n}(*)=\left\{\begin{array}{ll}
K, & \text { for } n=0, \\
0, & \text { for } n \geqslant 1,
\end{array} \text { and } \widetilde{H}_{n}(*)=0 \forall n \in \mathbb{Z} .\right.
$$

We have natural isomorphisms

$$
H_{*}(X, *) \simeq \widetilde{H}_{*}(X)
$$

and

$$
H_{n}(X, *) \simeq H_{n}(X) \text { for } n \geqslant 1 \text {. }
$$

Proof. The first statement follows directly from definition of path homology groups. The next statement follows from Proposition 3.14 and definition of relative homology groups (see also $[\mathbf{2 7}, \S 3.2]$ ).

Now we give a digraph interpretation of the relative homology groups $H_{*}(X, Y)$ and introduce the relative homology groups $H_{*}(f)$ for a digraph map $f: X \rightarrow X^{\prime}$. 
Theorem 3.18. For a digraph map $f: X \rightarrow X^{\prime}$ there is a homology long exact sequence

$$
\cdots \longrightarrow H_{n}(X) \stackrel{f_{*}}{\longrightarrow} H_{n}\left(X^{\prime}\right) \longrightarrow H_{n}\left(C_{f}, X\right) \longrightarrow H_{n-1}(X) \longrightarrow \cdots,
$$

where we identify $X$ with $j(X) \subset C_{f}$ by means of the natural inclusion $j: X \subset C_{f}$ from Definition 2.10.

Proof. Consider a commutative diagram of digraphs and digraph maps

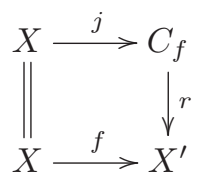

in which $r$ is the deformation retraction. From (9) we obtain the commutative diagram of groups and homomorphisms

$$
\begin{gathered}
\cdots \longrightarrow H_{n}(X) \stackrel{j_{*}}{\longrightarrow} H_{n}\left(C_{f}\right) \longrightarrow H_{n}\left(C_{f}, X\right) \longrightarrow \cdots \\
\cong r_{*} \\
H_{n}(X) \stackrel{f_{*}}{\longrightarrow} H_{n}\left(X^{\prime}\right)
\end{gathered}
$$

and the statement of the theorem follows.

In what follows we shall denote by $H_{*}(f)$ the relative homology groups $H_{*}\left(C_{f}, X\right)$. Note that there is a result that is similar to Theorem 3.18 for reduced homology groups. It is an easy exercise to reformulate the result for the inverse cylinder $C_{f}^{-}$.

Proposition 3.19. Let $Y \sqsubset X$ and assume that there is a deformation retraction $r: Y \rightarrow * \subset Y$. Then we have isomorphisms of relative homology groups

$$
H_{*}(X, Y) \cong H_{*}(X, *) \text {. }
$$

Proof. The natural map $(X, *) \rightarrow(X, Y)$ of digraph pairs induces the commutative diagram



Now the result follows from Theorem 3.15 and the Five Lemma, since the maps induced by $i$ and the identity map Id are isomorphisms.

Corollary 3.20. For any inclusion $i: Y \rightarrow X$ of digraphs we have isomorphisms of relative homology groups

$$
H_{*}\left(\text { Cone }_{i}, \text { Cone }(Y)\right) \cong H_{*}\left(\text { Cone }_{i}, *\right) .
$$

Proof. Any inclusion $i$ induces the inclusion $C o n e(Y) \subset C C_{i}$. Now the result follows from Proposition 3.19, since there is a deformation retraction of $C o n e(Y)$ onto its vertex $*$. 
Now consider a triple of digraphs $Z \sqsubset Y \sqsubset X$. Denote the inclusions of the digraphs in the following way

$$
i: Y \rightarrow X, j: Z \rightarrow Y, k=i \circ j: Z \rightarrow X .
$$

Theorem 3.21. For a triple of digraphs $Z \sqsubset Y \sqsubset X$ there is the commutative diagram of abelian groups and homomorphisms

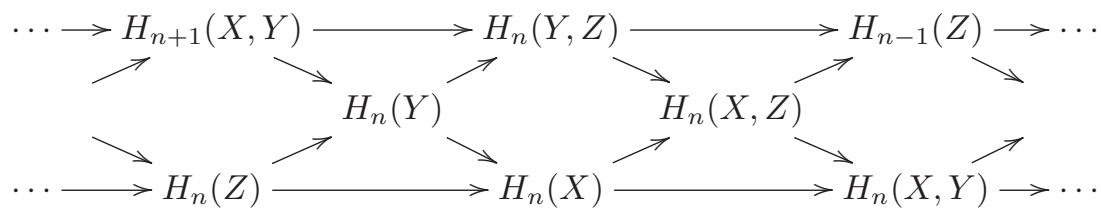

consisting of the following relative long exact sequences

$$
\begin{aligned}
& \cdots \longrightarrow H_{n+1}(X, Y) \longrightarrow H_{n}(Y) \stackrel{i_{*}}{\longrightarrow} H_{n}(X) \longrightarrow H_{n}(X, Y) \rightarrow \cdots, \\
& \cdots \longrightarrow H_{n+1}(X, Z) \longrightarrow H_{n}(X, Z) \rightarrow \cdots, \\
& \cdots \longrightarrow H_{n}(Z) \stackrel{k_{*}}{\longrightarrow} H_{n}(X) \longrightarrow H_{n+1}(Y, Z) \longrightarrow H_{n}(Z) \stackrel{j_{*}}{\longrightarrow} H_{n}(Y) \longrightarrow \cdots, \\
& \cdots \longrightarrow H_{n+1}(X, Y) \longrightarrow H_{n}(Y, Z) \longrightarrow H_{n}(X, Z) \longrightarrow H_{n}(X, Y) \rightarrow \cdots
\end{aligned}
$$

Proof. By Proposition 3.9, there are the natural inclusions of chain complexes

$$
\Omega_{*}(Z) \longrightarrow \Omega_{*}(Y) \longrightarrow \Omega_{*}(X) .
$$

By the Noether isomorphism theorem (see [30, Chpt. 4]) there is a short exact sequence of chain complexes

$$
0 \longrightarrow \Omega_{*}(Y) / \Omega_{*}(Z) \longrightarrow \Omega_{*}(X) / \Omega_{*}(Z) \longrightarrow \Omega_{*}(X) / \Omega_{*}(Y) \longrightarrow 0 .
$$

Thus we can write down the commutative diagram of chain complexes and chain homomorphisms

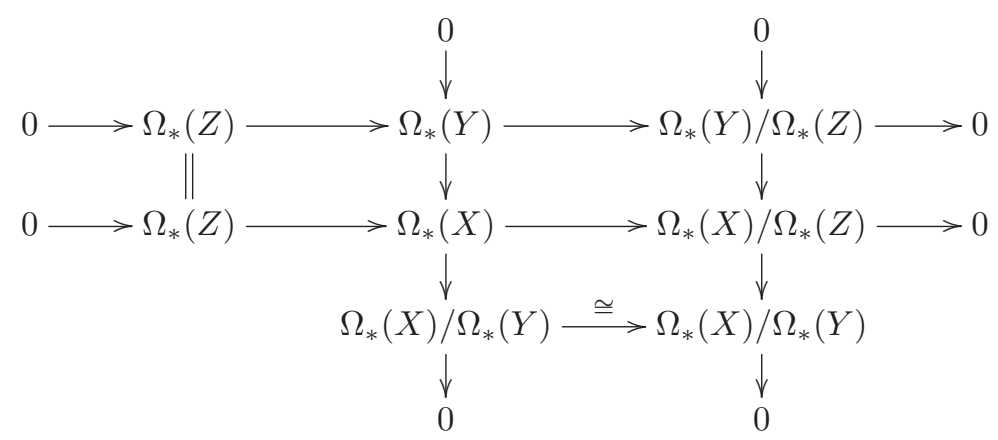

in which two rows and two columns are short exact sequences. Now, passing to the homology long exact sequences of these short exact sequences of chain complexes we obtain commutative diagram (10).

As follows from the proof, there exists the braid of exact sequence (10) also for reduced homology groups of the triple of digraphs. 
Corollary 3.22. Assume that, for a triple of digraphs $Z \sqsubset Y \sqsubset X$, one of the inclusions $i: Y \rightarrow X$ or $j: Z \rightarrow Y$ is a homotopy equivalence. Then in the first case we have an isomorphism $H_{*}(Y, Z) \cong H_{*}(X, Z)$, and in the second case $H_{*}(X, Z) \cong$ $H_{*}(X, Y)$.

Proof. Follows from Theorem 3.11 and diagram (10).

Note that the braid of exact sequences (10) is natural relative to digraph maps of triples of digraphs.

Now for the path homology groups, we formulate and prove a result that is similar to the classical Mayer-Vietoris exact sequence.

Let $X=Y_{1} \cup Y_{2}$ and $Z=Y_{1} \cap Y_{2}$ be, respectively, the union and intersection of two digraphs. Then we can write down the following commutative diagram of the natural inclusions of the digraphs:

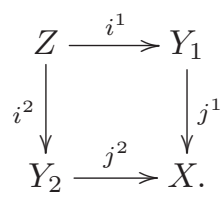

For any $p \geqslant-1$ the commutative square (11) induces a commutative square of abelian groups

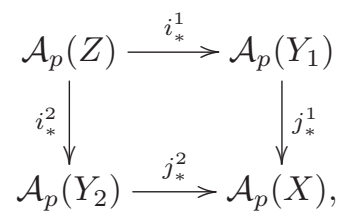

in which all homomorphisms are injective.

Lemma 3.23. Suppose that any allowed elementary path on $X$ lies in $Y_{1}$ or $Y_{2}$. Then for $p \geqslant-1$ the square (12) induces the following short exact sequence of abelian groups:

$$
0 \longrightarrow \mathcal{A}_{p}(Z) \stackrel{\delta}{\longrightarrow} \mathcal{A}_{p}\left(Y_{1}\right) \oplus \mathcal{A}_{p}\left(Y_{2}\right) \stackrel{d}{\longrightarrow} \mathcal{A}_{p}(X) \longrightarrow 0,
$$

where $\delta=\left(i_{*}^{1}, i_{*}^{2}\right)$ and

$$
d(a, b)=j_{*}^{1}(a)-j_{*}^{2}(b)
$$

Proof. The map $\delta$ is evidently a monomorphism. The map $d$ is an epimorphism by the assumptions. The condition $d \circ \delta=0$ follows from the definition of these maps and commutativity of (12). The condition $d(a, b)=0$ implies that the paths $j_{*}^{1}(a)$ and $j_{*}^{2}(b)$ coincide in $\mathcal{A}_{p}(X)$, and since the maps $j_{*}^{1}$ and $j_{*}^{2}$ are monomorphisms, this implies that elementary allowed $p$-paths fitting into basis decompositions of $a$ and $b$ are the same and hence these elementary $p$-paths lie in $\mathcal{A}_{p}\left(Y_{1} \cap Y_{2}\right)=\mathcal{A}_{p}(Z)$. This means that $\operatorname{Ker} d \subset \operatorname{Im} \delta$, and the lemma is proved. 
Lemma 3.24. Let the assumption of Lemma 3.23 be satisfied. Assume in addition that the homomorphism

$$
\Omega_{p}\left(Y_{1}\right) \oplus \Omega_{p}\left(Y_{2}\right) \longrightarrow \Omega_{p}(X)
$$

that is induced by drom Lemma 3.23 is an epimorphism for any $p$. Then there is a short exact sequence of chain complexes

$$
0 \longrightarrow \widetilde{\Omega}_{*}(Z) \stackrel{\delta}{\longrightarrow} \widetilde{\Omega}_{*}\left(Y_{1}\right) \oplus \widetilde{\Omega}_{*}\left(Y_{2}\right) \stackrel{d}{\longrightarrow} \widetilde{\Omega}_{*}(X) \longrightarrow 0 .
$$

Proof. It follows from Proposition 3.9 that there is a commutative diagram of chain complexes

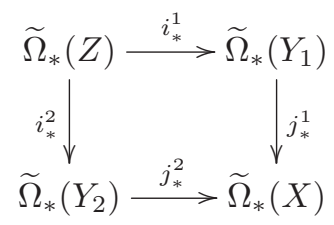

in which all maps are inclusions. Hence the map $\delta$ in (15) is a monomorphism. The map $d$ is an epimorphism by the assumptions of the lemma. From commutativity of (16) we obtain that $d \circ \delta=0$ in (13).

Now let $d(a, b)=j_{*}^{1}(a)-j_{*}^{2}(b)=0$ in $(13)$, where

$$
a \in \widetilde{\Omega}_{n}\left(Y_{1}\right) \subset \mathcal{A}_{n}\left(Y_{1}\right), b \in \widetilde{\Omega}_{n}\left(Y_{2}\right) \subset \mathcal{A}_{n}\left(Y_{2}\right) .
$$

By Lemma 3.23, there is an element $x \in \mathcal{A}_{n}(Z)$ such that

$$
i_{*}^{1}(x)=a, i_{*}^{2}(x)=b, \quad j_{*}^{1} \circ i_{*}^{1}(x)=j_{*}^{2} \circ i_{*}^{2}(x) \in \mathcal{A}_{n}(X) .
$$

Since all maps in (12) are inclusions, we conclude that $x \in \widetilde{\Omega}_{n}\left(Y_{1}\right)$ and $x \in \widetilde{\Omega}_{n}\left(Y_{2}\right)$. Hence $\partial x \in \mathcal{A}_{n-1}\left(Y_{1}\right)$ and $\partial x \in \mathcal{A}_{n-1}\left(Y_{2}\right)$, that is $\partial x \in \mathcal{A}_{n-1}(Z)$. Hence $x \in \widetilde{\Omega}_{n}(Z)$ and lemma is proved.

Theorem 3.25. (Mayer-Vietoris exact sequence) Under the assumptions of Lemma 3.24 the square (10) induces a long exact sequence of homology groups:

$$
\cdots \longrightarrow \widetilde{H}_{n}(Z) \stackrel{\delta}{\longrightarrow} \widetilde{H}_{n}\left(Y_{1}\right) \oplus \widetilde{H}_{n}\left(Y_{2}\right) \stackrel{d}{\longrightarrow} \widetilde{H}_{n}(X) \stackrel{\partial}{\longrightarrow} \widetilde{H}_{n-1}(Z) \longrightarrow \cdots,
$$

where $\delta=\left(i_{*}^{1}, i_{*}^{2}\right), d(a, b)=j_{*}^{1}(a)-j_{*}^{2}(b)$, and $\partial$ is a connecting homomorphisms.

Proof. Follows from Lemma 3.24 by the zig-zag lemma.

Note that under the assumptions of Lemma 3.24 there is also the similar MayerVietoris exact sequence of non-reduced homology groups.

Now we give yet one example in which the Mayer-Vietoris exact sequence exists.

Definition 3.26. Let $\left(Y_{1}, y_{1}\right)$ and $\left(Y_{2}, y_{2}\right)$ be based digraphs. The wedge sum (or bouquet)

$$
X=Y_{1} \bigvee_{y_{1} \sim y_{2}} Y_{2}
$$

of these digraphs is a digraph $X$ with the set $V_{X}$ of vertices that is obtained from the disjoint union $V_{Y_{1}} \cup V_{Y_{2}}$ by identification of based vertices $y_{1}$ and $y_{2}$, and with 
the set of edges $E_{X}=E_{Y_{1}} \cup E_{Y_{2}}$. We shall denote by $*$ the vertex that is obtained by identification of $y_{1}$ and $y_{2}$.

Identifying $Y_{i}$ with the sub-digraph of $X$ and denoting $*=Z$, we have natural inclusions of digraphs as in commutative diagram (11).

Lemma 3.27. Let $X=Y_{1} \bigvee_{y_{1} \sim y_{2}} Y_{2}$ and $Z=* \subset X$. Then there is a short exact sequence of chain complexes (15) and hence the Mayer-Vietoris exact sequence as in Theorem 3.25.

Proof. We have the commutative square (16) in which all the maps are inclusions. Hence the map $\delta$ is a monomorphism and it follows from the commutativity that $d \circ \delta=0$.

Let us show that $\operatorname{Ker} d \subset \operatorname{Im} \delta$. If $\omega \in \operatorname{Ker} d$ then $\omega=(a, b)$ where $a \in \widetilde{\Omega}_{*}\left(Y_{1}\right)$, $b \in \widetilde{\Omega}_{*}\left(Y_{2}\right)$ and $j_{*}^{1}(a)=j_{*}^{2}(b)$. However, the latter is possible only if $a$ and $b$ have dimensions 0 or -1 . For these two cases the result follows directly from (16).

Finally, let us check that $d$ in (15) is an epimorphism. In the dimensions $-1,0,1$ this is evidently. If $p \geqslant 2$ and $\omega \in \widetilde{\Omega}_{*}(X)$ then consider an elementary term $c e_{i_{0} \ldots i_{p}}$ of $\omega(c \neq 0)$. We need only to show that all the vertices $i_{0} \ldots i_{p}$ lie either in $Y_{1}$ or in $Y_{2}$. Indeed, assume that this is not the case, that is, $*$ is one of the vertices $i_{1}, \ldots, i_{p-1}$. The path $\partial\left(c e_{i_{0} \ldots i_{p}}\right)$ contains the term $c e_{i_{0} \ldots \widehat{*} \ldots i_{p}}$ that is not allowed. This term must be cancelled in $\partial \omega$ using another elementary term of $\omega$. However, the latter term should then be $-c e_{i_{0} \ldots i_{p}}$, which is not possible.

Corollary $\mathbf{3 . 2 8}$ (Cf. [17, Th.4.17]). Under assumptions of Lemma 3.27 we have isomorphisms

$$
\widetilde{H}_{n}\left(Y_{1} \bigvee Y_{2}, K\right) \cong \widetilde{H}_{n}\left(Y_{1}, K\right) \oplus \widetilde{H}_{n}\left(Y_{2}, K\right)
$$

Proof. Follows from the Mayer-Vietoris exact sequence for $*, Y_{1}, Y_{2}, Y_{1} \bigvee Y_{2}$ and the isomorphisms $\widetilde{H}_{*}(*, K)=0$ in Proposition 3.17.

Now we give the path homology version of the excision axiom of Eilenberg-Steenrod.

Theorem 3.29. Let $X=\left(V_{X}, E_{X}\right)$ be a digraph in which there are two subsets $U_{1}, U_{2}$ of $V_{X}$ such that there are no outcoming arrows from $U_{i}$ to $V_{X} \backslash U_{i}$ for $i=1,2$. Denote by $Y_{i}(i=1,2)$ the digraph that is obtained from $X$ by deleting all the vertices from $U_{k}$ with $k \neq i$ and all arrows adjoint to these vertices (see Fig. 1). Then the inclusion

$$
j:\left(Y_{1}, Y_{1} \cap Y_{2}\right) \rightarrow\left(X, Y_{2}\right)
$$

induces an isomorphism of chain complexes

$$
j_{*}: \Omega_{*}\left(Y_{1}\right) / \Omega_{*}\left(Y_{1} \cap Y_{2}\right) \rightarrow \Omega_{*}(X) / \Omega_{*}\left(Y_{2}\right)
$$

and, hence, an isomorphism of homology groups

$$
j_{*}: H_{n}\left(Y_{1}, Y_{1} \cap Y_{2}\right) \rightarrow H_{n}\left(X, Y_{2}\right) .
$$




$$
X=Y_{1} \cup Y_{2}
$$

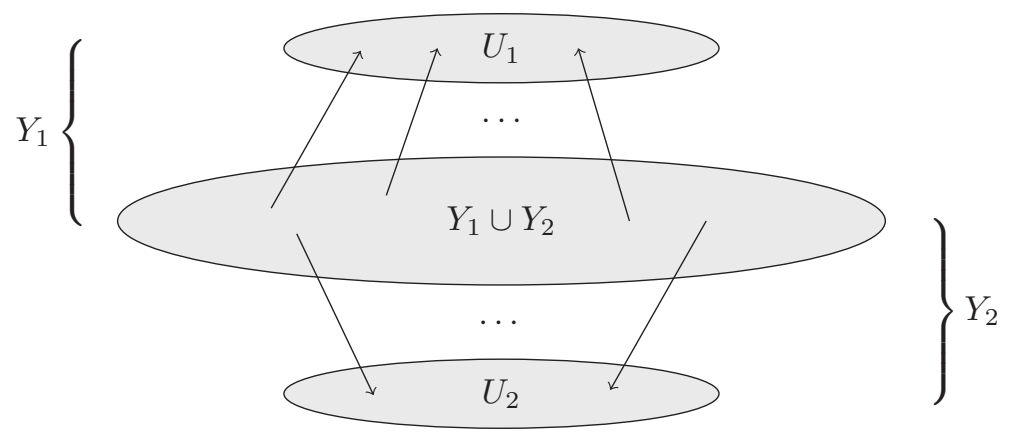

Figure 1: Digraphs $X, Y_{1}, Y_{2}$.

Proof. By Lemma 3.10 we have isomorphisms

$$
\Omega_{n}(X) / \Omega_{n}\left(Y_{2}\right) \cong \Omega_{n}^{U_{1}}(X)
$$

and

$$
\Omega_{n}\left(Y_{1}\right) / \Omega_{n}\left(Y_{1} \cap Y_{2}\right) \cong \Omega_{n}^{U_{1}}\left(Y_{1}\right) .
$$

The inclusion $j$ induces a morphism $j_{*}$ of chain complexes, so that we have the following commutative diagram:

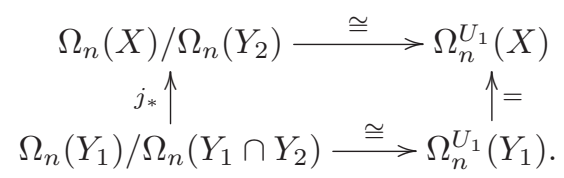

Here the homomorphism $\Omega_{n}^{U_{1}}(X) \rightarrow \Omega_{n}^{U_{1}}\left(Y_{1}\right)$ is induced by the inclusion and the fact that it is the identity follows from the hypothesis that there are no outcoming arrows from $U_{2}=V_{X} \backslash V_{Y_{1}}$ to $V_{Y_{1}}$. Hence, $j_{*}$ is an isomorphism of chain complexes, which was to be proved.

Note that a similar result to Theorem 3.29 is true if we change in this theorem "outcoming arrows" to "incoming arrows".

\section{Homotopy constructions: cylinder, cone and suspension}

In this section we give several new constructions of digraphs which are very helpful for developing path homology theory. In particular, we describe several transformations of digraphs that preserve homology groups.

Definition 4.1. For any digraph $X$ and any line digraph $I_{n} \in \mathcal{I}_{n}$ define a direct cylinder of $X$ as the digraph

$$
\operatorname{Cyl}^{d}(X)=X \square I_{n}, \quad I_{n} \in \mathcal{I}_{n}, n \geqslant 1 .
$$

Note that there exist $2^{n}$ digraphs $X \square I_{n}$ and hence, the cylinder is not unique. 
It is easy to check that there is a strong deformation retraction $C y l^{d}(X) \rightarrow X$ (see $[\mathbf{1 6}]$ ), and hence we have a homotopy equivalence $C y l^{d}(X) \simeq X$.

Definition $4.2([\mathbf{1 6}])$. A digraph map $h: I_{n} \rightarrow I_{m}$ is called shrinking if $h(0)=0$, $h(n)=m$, and $h(i) \leqslant h(j)$ whenever $i \leqslant j$.

Any shrinking map $h: I_{n} \rightarrow I_{m}$ is surjective and the preimage of any arrow of $I_{m}$ consists of exactly one arrow of $I_{n}$. Furthermore, we have necessarily $m \leqslant n$, and if $n=m$ then $h$ is a bijection.

Let $h: I_{n} \rightarrow I_{m}$ be a shrinking map. Then the cylinder $C_{h}$ and inverse cylinder $C_{h}^{-}$of the map $h$ were defined in Definition 2.10. Define a double cylinder $C_{h h}$ of the map $h$ as the following digraph:

$$
C_{h h}=C_{h} \cup C_{h}^{-}, \quad \text { where } V_{C_{h h}}=V_{I_{n}} \cup V_{I_{m}}
$$

and

$$
E_{C_{h h}}=E_{I_{n}} \cup E_{I_{m}} \cup\left\{i \rightarrow h(i) \mid i \in V_{I_{n}}\right\} \cup\left\{i \leftarrow h(i) \mid i \in V_{I_{n}}\right\} .
$$

Fix a digraph $X=\left(V_{X}, E_{X}\right)$. We write $v \sim w$ if $v$ and $w$ are adjacent vertices of $X$. In order to define a homotopy cylinder of $X$ we need the following preliminary construction:

i) Choose a collection $C(V)=\{I(v)\}_{v \in V}$ where each $I(v)$ is a line digraph from $\mathcal{I}_{n(v)}$ with $n(v) \geqslant 0$.

ii) For any pair $v \sim w$ we choose either a shrinking map $h_{v, w}: I(v) \rightarrow I(w)$ or a shrinking map $h_{w, v}: I(w) \rightarrow I(v)$.

iii) For any pair $v \sim w$ we define a digraph $C_{v \sim w}$ as follows. In the case $(v \rightarrow w) \in$ $E_{X},(w \rightarrow v) \notin E_{X}$ we set

$$
C_{v \sim w}= \begin{cases}C_{h_{v, w}} & \text { if } h_{v, w} \text { was chosen in ii), } \\ C_{h_{w, v}}^{-} & \text {if } h_{w, v} \text { was chosen in ii). }\end{cases}
$$

In the case $(v \rightarrow w) \in E_{X},(w \rightarrow v) \in E_{X}$ set

$$
C_{v \sim w}= \begin{cases}C_{h_{v, w} h_{v, w}} & \text { if } h_{v, w} \text { was chosen in ii), } \\ C_{h_{w, v} h_{w, v}} & \text { if } h_{w, v} \text { was chosen in ii). }\end{cases}
$$

Definition 4.3. Assume that $X$ is a connected digraph and $E_{X} \neq \emptyset$. A homotopy cylinder $C y l^{h}(X)$ of $X$ is a digraph that is given by the union

$$
\bigcup_{v \sim w} C_{v \sim w}
$$

where we identify the sub-digraphs $I(v)$ lying in various digraphs $C_{v \sim w}$ in a natural way.

By definition, we have natural inclusions

$$
i_{v}: I(v) \rightarrow C y l^{h}(X) \text { and } j_{v w}: C_{v \sim w} \rightarrow C y l^{h}(X),
$$

for any vertex $v \in V_{X}$ and for any pair $v \sim w$.

Note that any direct cylinder $C y l^{d}(X)$ has a natural structure of homotopy cylinder. For the next statements, we always assume that $X$ is connected and $E_{X} \neq \emptyset$. 
Proposition 4.4. For a homotopy cylinder $C y l^{h}(X)$ there are natural inclusions

$$
i_{0}: X \longrightarrow C y l^{h}(X) \text { and } i_{1}: X \longrightarrow C y l^{h}(X)
$$

and a retraction

$$
r: C y l^{h}(X) \longrightarrow X
$$

such that $r \circ i_{0}=\operatorname{Id}_{X}, r \circ i_{1}=\operatorname{Id}_{X}$.

Proof. Define an inclusion $i_{0}: X \rightarrow C y l^{h}(X)$ on the set of vertices by the composition

$$
v \stackrel{v \rightarrow 0}{\longrightarrow} I(v) \stackrel{i_{v}}{\longrightarrow} C y l^{h}(X),
$$

where the first map is the inclusion $v \rightarrow 0 \in I(v)$. Define an inclusion $i_{1}: X \rightarrow C y l^{h}(X)$ on the set of vertices by the composition

$$
v \stackrel{v \rightarrow n(v)}{\longrightarrow} I(v) \stackrel{i_{v}}{\longrightarrow} C y l^{h}(X),
$$

where the first map is the inclusion $v \rightarrow n(v) \in I(v)$, where $n(v)=|I(v)|$. Define the map $r$ on the set of vertices by $r(v, k)=v$ for $v \in V_{X}, k \in V_{I(v)}$.

Theorem 4.5. The map $r$ in Proposition 4.4 is a strong deformation retraction.

Proof. Denote by $C$ the digraph $C y l^{h}(X)$. If all $n(v)=0$ then there is nothing to prove. Consider a vertex $v \in V_{X}$ with $n(v) \geqslant 1$. Then $(v, n(v)-1) \sim(v, n(v))$ in $C$.

Consider a set $W$ of all vertices $w$ of $X$ that satisfy the following property: there is a sequence of vertices $v=v_{0}, v_{1}, \ldots, v_{s}=w$ on $X$ such that $v_{i} \sim v_{i+1}$ in $X$ for any $i=0, \ldots, s-1$, and

$$
\left(v_{i}, n\left(v_{i}\right)\right) \sim\left(v_{i+1}, n\left(v_{i+1}\right)\right),\left(v_{i}, n\left(v_{i}\right)-1\right) \sim\left(v_{i+1}, n\left(v_{i+1}\right)-1\right),
$$

in $C$. Define a digraph map $\phi: C \rightarrow C$ on the set of vertices of $C$ by

$$
\phi(u, k)= \begin{cases}(u, k-1), & \text { for }(u, k)=(w, n(w)), \quad w \in W \cup\{v\}, \\ (u, k), & \text { otherwise. }\end{cases}
$$

The map $\phi$ is a strong deformation retraction (see [16, Corollary 3.7]) of $C$ to a sub-digraph that is also a homotopical cylinder over $X$ and that has $\left|V_{C}\right|-|W|-1$ vertices. From now the result follows by induction using Proposition 2.8.

Corollary 4.6. For any finite digraph $X$ and any homotopy cylinder digraph $C_{y l}^{h}(X)$ we have an isomorphism

$$
\widetilde{H}_{*}(X) \cong \widetilde{H}_{*}\left(C y l^{h}(X)\right) .
$$

Proof. Follows from Theorem 3.8 .

Now we introduce a new definition of homotopy and prove that it is equivalent to our previous definition. As we shall see below this generalization is very natural.

Definition 4.7. Let $f, g: X \rightarrow Y$ be digraph maps. A digraph map $F: C y l^{h}(X) \rightarrow Y$ is called a generalized homotopy of $f$ and $g$ if

$$
F \circ i_{0}=f, \quad F \circ i_{1}=g,
$$

where $i_{0}, i_{1}$ are the inclusions from Proposition 4.4. In this case the maps $f$ and $g$ are called generalized homotopic. 
Theorem 4.8. Let $X$ be a finite digraph. Two digraph maps $f, g: X \rightarrow Y$ are generalized homotopic if and only if they are homotopic.

Proof. In one direction the statement is trivial since a homotopy provides evidently a generalized homotopy.

Now by Theorem 4.5 there is a strong deformation retraction

$$
R: C y l^{h}(X) \square I_{n} \rightarrow C y l^{h}(X)
$$

such that

$$
\left.R\right|_{C y l^{h}(X) \square\{0\}}=\mathrm{Id},\left.\quad R\right|_{C y l^{h}(X) \square\{n\}}: C y l^{h}(X) \square\{n\} \rightarrow i_{0}(X),
$$

where the last map is the identity on $i_{0}(X)$, and as follows from the proof of Theorem 4.5 it maps isomorphically the sub-digraph $i_{1}(X)$ to $i_{0}(X)$ (for any vertex $v \in V_{X}$ we have $\left.R\left(i_{1}(v)\right)=i_{0}(v)\right)$.

Let $F$ be a generalized homotopy of $f$ and $g$. Consider a composition $\Psi$ of digraph maps

$$
X \square I_{n} \stackrel{i \square \mathrm{Id}}{\longrightarrow} i_{1}(X) \square I_{n} \stackrel{R}{\longrightarrow} C y l^{h}(X) \stackrel{F}{\longrightarrow} Y,
$$

where $\left.\Psi\right|_{X \square\{0\}}=F \circ i_{1}(X)=g$ and $\left.\Psi\right|_{X \square\{n\}}=F \circ i_{0}(G)=f$. Clearly, $\Psi$ is a homotopy of $f, g$.

Corollary 4.9. Generalized homotopy digraph maps induce the same homomorphism of (reduced) path homology groups.

Definition 4.10. i) Define a homotopy cone $C_{0 n e}(X)$ of a digraph $X$ as a digraph that is obtained from a homotopy cylinder $C y l^{h}(X)$ by adding a new vertex $a$ and the set of arrows

$$
\left\{(v, n(v)) \rightarrow a \mid \forall v \in V_{X}\right\} .
$$

ii) Define an inverse homotopy cone $C_{0 n} e^{h^{-}}(X)$ of a digraph $X$ as a digraph that is obtained from a homotopy cylinder $C y l^{h}(X)$ by adding a new vertex $a$ and the set of arrows

$$
\left\{(v, n(v)) \leftarrow a \mid \forall v \in V_{X}\right\} .
$$

The vertex $a$ will be referred to as a vertex of the (inverse) homotopy cone.

Note, that the cone $C o n e(X)$ and the inverse cone $C o n e^{-}(X)$ of a digraph $X$ (see Definition 2.9) are particular cases of a homotopy cone and inverse homotopy cone, respectively. It follows directly from Definition 4.10, that there are natural inclusions $i: X \rightarrow \operatorname{Cone}^{h}(X)$ and $i^{-}: X \rightarrow$ Cone $^{h^{-}}(X)$ that are induced by the inclusion $i_{0}: X \rightarrow C y l^{h}(X)$. Using these inclusions we can consider the digraph $X$ as a subdigraph of $\operatorname{Cone}^{h}(X)$ and $\operatorname{Cone}^{h^{-}}(X)$.

Theorem 4.11. Let $X$ be a digraph and let $\operatorname{Cone}^{h}(X)$ be a homotopy cone with a vertex $*$. Then there are strong deformation retractions

$$
r: \operatorname{Cone}^{h}(X) \longrightarrow *, r^{-}: \operatorname{Cone}^{h^{-}}(X) \longrightarrow *,
$$

and hence, in particular, $\operatorname{Cone}^{h}(X) \simeq * \simeq \operatorname{Cone}^{h^{-}}(X)$.

Proof. Similar to Theorem 4.5. 
Definition 4.12. The homotopy suspension $S^{h}(X)$ of a digraph $X$ is a digraph defined as a union of two not necessarily equal digraphs of $\operatorname{Cone}_{1}^{h}(X)$ and $\operatorname{Cone}_{2}^{h}(X)$ that intersect in $X$ :

$$
S^{h}(X)=\operatorname{Cone}_{1}^{h}(X) \bigcup_{X} \operatorname{Cone}_{2}^{h}(X)
$$

The inverse homotopy suspension $S^{h^{-}}(X)$ of a digraph $X$ is a digraph defined as a union of two not necessarily equal digraphs of $\operatorname{Cone}_{1}^{h^{-}}(X)$ and $\operatorname{Cone}_{2}^{h^{-}}(X)$ that intersect in $X$ :

$$
S^{h^{-}}(X)=\text { Cone }_{1}^{h^{-}}(X) \bigcup_{X} \operatorname{Cone}_{2}^{h^{-}}(X) .
$$

Note that the suspension $S(X)$ and inverse suspension $S^{-}(X)$ of a digraph $X$ (see Definition 2.12) are particular cases of homotopy suspensions.

Now we would like to describe homology properties of homotopy suspensions.

Theorem 4.13. Let $X$ be a digraph. Then for any $n \geqslant 0$ there is an isomorphism $\widetilde{H}_{n+1}(S(X)) \cong \widetilde{H}_{n}(X)$.

Proof. By Definition 2.12 we have

$$
S(X)=Y_{1} \bigcup_{X} Y_{2}
$$

where $Y_{i}=$ Cone $_{a_{i}}(X), i=1,2$ with distinct vertices $a_{1}, a_{2}$. Observe that any allowed elementary path $x$ on $S(X)$ lies in $Y_{1}$ or $Y_{2}$, so that the hypothesis of Lemma 3.23 is satisfied. Let us verify also the hypothesis (14) of Lemma 3.24, that is, any $\partial$ invariant path on $S(X)$ is a sum of $\partial$-invariant paths on $Y_{1}$ and $Y_{2}$. Clearly, any path $x \in \mathcal{A}_{n}(S(X))$ can be represented in the form

$$
x=w+w_{1}+w_{2},
$$

where $w \in \mathcal{A}_{n}(X), w_{1} \in \mathcal{A}_{n}^{\left\{a_{1}\right\}}\left(Y_{1}\right), w_{2} \in \mathcal{A}_{n}^{\left\{a_{2}\right\}}\left(Y_{2}\right)$ (in the notation of Lemma 3.10). Let now $x \in \Omega_{n}(S(X))$ so that $\partial x \in \mathcal{A}_{n-1}(S(X))$. Since $\partial x=\partial w+\partial w_{1}+\partial w_{2}$, arguing as in the proof of Lemma 3.10 we obtain that $\partial w \in \mathcal{A}_{n-1}(X)$ and $\partial w_{i}=u_{i}+v_{i}$ where $u_{i} \in \mathcal{A}_{n-1}(X)$ and $v_{i} \in \mathcal{A}_{n-1}^{\left(a_{i}\right)}\left(Y_{i}\right)$. Consequently, $w \in \Omega_{n}(X), w_{i} \in \Omega_{n}\left(Y_{i}\right)$.

Hence, any $x \in \Omega_{n}(S(X))$ can be written in the form $x=\left(w+w_{1}\right)+w_{2}$ where

$$
\left(w+w_{1}\right) \in \Omega_{n}\left(Y_{1}\right) \text { and } w_{2} \in \Omega_{n}\left(Y_{2}\right) .
$$

By Lemma 3.24 we obtain the Mayer-Vietoris exact sequence of reduced homology groups

$$
\cdots \rightarrow \widetilde{H}_{n}(X) \rightarrow \widetilde{H}_{n}\left(Y_{1}\right) \oplus \widetilde{H}_{n}\left(Y_{2}\right) \rightarrow \widetilde{H}_{n}(S(X)) \rightarrow \widetilde{H}_{n-1}(X) \rightarrow \cdots .
$$

The cone $Y_{i}$ is homotopy equivalent to a one-vertex digraph, hence all reduced homology groups of $Y_{i}$ are trivial. Now from the exact sequence we obtain the claim.

Theorem 4.14. For any finite digraph $X$ and for $n \geqslant 0$ there are isomorphisms

$$
\widetilde{H}_{n+1}\left(S^{h}(X)\right) \cong \widetilde{H}_{n}(X),
$$

where $S^{h}(X)$ is a homotopy suspension of the digraph $X$.

Proof. The proof is similar to Theorem 4.13. 


\section{Path homology theory and Eilenberg-Steenrod axioms}

In this section we present an axiomatic approach to the definition of path homology theory on the category of digraph pairs. The situation here is very similar to the classical Eilenberg-Steenrod axioms of algebraic topology (see, for example, $[\mathbf{2 6}, \S 26]$ ).

Denote by $R$ a functor $\mathcal{D}^{2} \rightarrow \mathcal{D}^{2}$ defined by the rule

$$
R(X, Y)=(Y, \emptyset) \text { and } R(f)=\left.f\right|_{Y}\left(f:(X, Y) \rightarrow\left(X^{\prime}, Y^{\prime}\right)\right) .
$$

This functor evidently induces the functor $\operatorname{Ho} R: \operatorname{Ho}^{2} \rightarrow$ Ho $\mathcal{D}^{2}$ by the rule

$$
(\operatorname{Ho} R)(X, Y)=(Y, \emptyset) \text { and }(\operatorname{Ho} R)[f]=\left[\left.f\right|_{Y}\right]\left(f:(X, Y) \rightarrow\left(X^{\prime}, Y^{\prime}\right)\right),
$$

where $[f]$ denote the homotopy class of the map $f$. Let $\mathcal{A}$ be the category of abelian groups and homomorphisms of abelian groups.

Definition 5.1. A non-reduced homology theory on the category Ho $\mathcal{D}^{2}$ of digraph pairs and homotopy classes of digraph maps of pairs consists of the sequence of functors

$$
H_{p}: \operatorname{Ho}^{2} \rightarrow \mathcal{A} \text { for } p \in \mathbb{Z}
$$

and natural transformations

$$
\partial_{n}: H_{n} \longrightarrow H_{n-1} \circ R \text { for } p \in \mathbb{Z}
$$

such that the following axioms are satisfied.

i) Exactness axiom: for every pair $(X, Y) \in H_{0} \mathcal{D}^{2}$ the sequence

$$
\cdots \rightarrow H_{n+1}(X, Y) \stackrel{\partial_{n+1}(X, Y)}{\longrightarrow} H_{n}(Y, \emptyset) \stackrel{H_{n}(i)}{\longrightarrow} H_{n}(X, \emptyset) \stackrel{H_{n}(j)}{\longrightarrow} H_{n}(X, Y) \stackrel{\partial_{n}(X, Y)}{\longrightarrow} \cdots
$$

is exact, where

$$
i:(Y, \emptyset) \rightarrow(X, \emptyset), \quad j:(X, \emptyset) \rightarrow(X, Y)
$$

are the natural inclusions.

ii) Excision axiom. Consider two cases.

(a) Let $X$ be a digraph in which there are two sub-digraphs $A_{1}$ and $A_{2}$ such that there are no outcoming arrows from $V_{A_{i}}$ to $V_{X} \backslash V_{A_{i}}$ for $i=1,2$. Let $Y_{i}(i=1,2)$ be obtained from $X$ by deleting the digraph $A_{i}$ and all incoming to $V_{A_{i}}$ arrows starting from $V_{X} \backslash V_{A_{i}}$. ("Outcoming arrows" can be replaced everywhere by "incoming arrows".)

(b) Let $X=Y_{1} \cup Y_{2}$ and $Y_{1} \cap Y_{2}=*$ is a one-vertex digraph.

In both cases (a), (b) the inclusion

$$
j:\left(Y_{1}, Y_{1} \cap Y_{2}\right) \rightarrow\left(X, Y_{2}\right)
$$

induces isomorphisms

$$
H_{n}(j): H_{n}\left(Y_{1}, Y_{1} \cap Y_{2}\right) \rightarrow H_{n}\left(X, Y_{2}\right), \quad n \in \mathbb{Z} .
$$

iii) Additivity axiom: if $X=\bigcup_{\alpha} X_{\alpha}$ is the disjoint union of digraphs then

$$
H_{n}(X, \emptyset)=\bigoplus_{\alpha} H_{n}\left(X_{\alpha}, \emptyset\right) .
$$


iv) Dimension axiom: for a one-vertex digraph $*$ we have

$$
H_{n}(*, \emptyset)= \begin{cases}K, & n=0, \\ 0, & n \neq 0,\end{cases}
$$

where $K$ is fixed abelian group.

Theorem 5.2. There exists a homology theory that satisfies this system of axioms.

Proof. The proof follows from the results of Section 3.

Remark 5.3. The question remains, though, of what additional axioms are necessary to ensure that the path homology theory is a unique realization.

\section{Examples}

In this section we consider several examples which illustrate the difference between the path homology theory and the homology theory for topological or simplicial spaces.

Example 6.1. i) Let $i: Y=* \cup \star \subset X$ where $X$ is the following digraph $* \leftarrow \bullet \rightarrow \star$. Then $H_{0}(Y)=K \oplus K, H_{0}(X)=K$ and other homology groups of these digraphs are trivial. Hence $H_{1}(X, Y)=K$ and $H_{1}(X, Y) \cong H_{1}\left(\right.$ Cone $\left._{i}\right)$.

ii) Let $i: Y=* \cup \star \subset X$ where $X$ is the following digraph $* \rightarrow \bullet \leftarrow \star$. Then $H_{0}(Y)=K \oplus K, H_{0}(X)=K$ and other homology groups of these digraphs are trivial. Hence $H_{1}(X, Y)=K$ and $H_{1}\left(\right.$ Cone $\left._{i}\right)=0$.

Example 6.2. i) Consider a digraph $X$

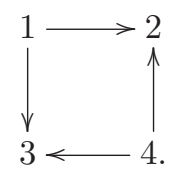

Taking one point sub-digraphs $A_{1}=\{2\}, A_{2}=\{3\}$ we can define sub-digraphs $Y_{1}$ and $Y_{2}$ and apply the excision axiom for computing $H_{p}(X)$. We obtain that $H_{p}(X) \cong K$ for $p=0,1$ and trivial for $p \geqslant 2$.

ii) Consider a digraph $X$



In this case we cannot cut $X$ in two parts for applying the excision axiom. However, the homology groups of this digraph are the same as those in the previous example.

\section{References}

[1] R.H. Atkin, An algebra for patterns on a complex, I. Int. J. Man-Mach. Stud. 6 (1974), 285-307. 
[2] R. Atkin, An algebra for patterns on a complex, II. Int. J. Man-Mach. Stud. 8 (1976), 483-498.

[3] E. Babson, H. Barcelo, M. de Longueville and R. Laubenbacher, Homotopy theory of graphs. J. Algebraic Combin. 24 (2006), 31-44.

[4] H.C. Baehr, A. Dimakis and F. Müller-Hoissen, Differential calculi on commutative algebras. J. Phys. A 28 (1995), 3197-3222.

[5] H. Barcelo, V. Capraro and J.A. White, Discrete homology theory for metric spaces. Bull. Lond. Math. Soc. 46 (2014), 889-905.

[6] H. Barcelo, X. Kramer, R. Laubenbacher and W. Christopher, Foundations of a connectivity theory for simplicial complexes. Adv. Appl. Math. 26 (2001), 97-128.

[7] B. Chen, S.-T. Yau and Y.-N. Yeh, Graph homotopy and Graham homotopy. Discrete Math. 241 (2001), 153-170.

[8] C. Cibils, Cohomology of incidence algebras and simplicial complexes. J. Pure Appl. Algebra 56 (1989), 221-232.

[9] A. Connes, Noncommutative Geometry, Academic Press, San Diego, 1994.

[10] A. Dimakis and F. Müller-Hoissen, Differential calculus and gauge theory on finite sets. J. Phys. A 27 (1994), 3159-3178.

[11] A. Dimakis and F. Müller-Hoissen, Discrete differential calculus: graphs, topologies, and gauge theory. J. Math. Phys. 35 (1994), 6703-6735.

[12] A. Dochtermann, Homotopy groups of Hom complexes of graphs. J. Combin. Theory Ser. A 116 (2009), 180-194.

[13] N.P. Dolbilin, M.A. Shtan'ko and M.I. Shtogrin, Cubic manifolds in lattices (in Russian); translated from Izv. Ross. Akad. Nauk Ser. Mat. 58 (1994), 93-107; Russian Acad. Sci. Izv. Math. 44 (1995), 301-313.

[14] N.P. Dolbilin, Yu.M. Zinov'ev, A.S. Mishchenko, M.A. Shtan'ko and M.I. Shtogrin, Homological properties of two-dimensional coverings of lattices on surfaces (in Russian); translated from Funktsional. Anal. i Prilozhen. 30 (1996), 19-33, 95; Funct. Anal. Appl. 30 (1996), 163-173 (1997).

[15] M. Gerstenhaber and S.D. Schack, Simplicial cohomology is Hochschild cohomology. J. Pure Appl. Algebra 30 (1983), 143-156.

[16] A. Grigor'yan, Y. Lin, Y. Muranov and S.-T. Yau, Homotopy theory for digraphs. Pure Appl. Math. Q. 10 (2014), 619-674.

[17] A. Grigor'yan, Y. Lin, Y. Muranov and S.-T. Yau, Cohomology of digraphs and (undirected) graphs. Asian J. Math. 19 (2015), 887-932.

[18] A. Grigor'yan, Y. Muranov and S.-T. Yau, Graphs associated with simplicial complexes. Homology Homotopy Appl. 16 (2014), 295-311.

[19] A. Grigor'yan, Y. Muranov and S.-T. Yau, On a cohomology of digraphs and Hochschild cohomology. J. Homotopy Relat. Struct. 11 (2016), 209-230.

[20] A. Hatcher, Algebraic Topology, Cambridge University Press, Cambridge, 2002.

[21] M. Herlihy, D. Kozlov and S. Rajsbaum, Distributed Computing through Combinatorial Topology, Elsevier/Morgan Kaufmann, Waltham, 2014. 
[22] G. Hochschild, On the cohomology groups of an associative algebra. Ann. of Math. (2) 46 (1945), 58-67.

[23] A.V. Ivashchenko, Contractible transformations do not change the homology groups of graphs. Discrete Math. 126 (1994), 159-170.

[24] D. Kozlov, Combinatorial Algebraic Topology, volume 21 of Algorithms Comput. Math., Springer, Berlin, 2008.

[25] S. MacLane, Homology, volume 114 of Grundlehren Math. Wiss., SpringerVerlag, Berlin, New York, 1967.

[26] J.R. Munkres, Elements of Algebraic Topology, Addison-Wesley Publishing Company, Menlo Park, 1984.

[27] V.V. Prasolov, Elements of Homology Theory, volume 81 of Grad. Stud. Math., American Mathematical Society, Providence, 2007.

[28] P. Ribenboim, Algebraic structures on graphs, Algebra Universalis 16 (1983), 105-123.

[29] M.A. Shtan'ko and M.I. Shtogrin, Embedding cubic manifolds and complexes into a cubic lattice (in Russian); translated from Uspekhi Mat. Nauk 47 (1992), 219-220; Russian Math. Surveys 47 (1992), 267-268.

[30] E.H. Spanier, Algebraic Topology, Springer-Verlag, New-York, 1995.

[31] M.E. Talbi and D. Benayat, Homology theory of graphs. Mediterr. J. Math. 11 (2014), 813-828.

[32] R.R. Zapatrin, Polyhedral representations of discrete differential manifolds. J. Math. Phys. 38 (1997), 2741-2750.

Alexander Grigor'yan grigor@math.uni-bielefeld.de

Mathematics Department, University of Bielefeld, Postfach 100131, Bielefeld, D33501, Germany

Institute of Control Sciences of Russian Academy of Sciences, Moscow, Russia

Rolando Jimenez rolando@matcuer. unam.mx

Instituto de Matematicas, UNAM, Unidad Oaxaca, Leon 2, Centro, Oaxaca, 68000, Mexico

Yuri Muranov muranov@matman.uwm.edu.pl

Faculty of Mathematics and Computer Science, University of Warmia and Mazury, Sloneczna 54 Street, Olsztyn, 10-710, Poland

Shing-Tung Yau yau@math.harvard.edu

Mathematics Department, Harvard University, One Oxford Street, Cambridge, MA 02138, USA 